\title{
Transient Topographical Dynamics of the Electroencephalogram Predict Brain Connectivity and Behavioural Responsiveness During Drowsiness
}

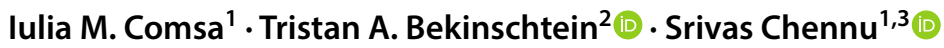

Received: 25 December 2017 / Accepted: 22 November 2018 / Published online: 29 November 2018

(c) The Author(s) 2018

\begin{abstract}
As we fall sleep, our brain traverses a series of gradual changes at physiological, behavioural and cognitive levels, which are not yet fully understood. The loss of responsiveness is a critical event in the transition from wakefulness to sleep. Here we seek to understand the electrophysiological signatures that reflect the loss of capacity to respond to external stimuli during drowsiness using two complementary methods: spectral connectivity and EEG microstates. Furthermore, we integrate these two methods for the first time by investigating the connectivity patterns captured during individual microstate lifetimes. While participants performed an auditory semantic classification task, we allowed them to become drowsy and unresponsive. As they stopped responding to the stimuli, we report the breakdown of alpha networks and the emergence of theta connectivity. Further, we show that the temporal dynamics of all canonical EEG microstates slow down during unresponsiveness. We identify a specific microstate (D) whose occurrence and duration are prominently increased during this period. Employing machine learning, we show that the temporal properties of microstate $\mathrm{D}$, particularly its prolonged duration, predicts the response likelihood to individual stimuli. Finally, we find a novel relationship between microstates and brain networks as we show that microstate D uniquely indexes significantly stronger theta connectivity during unresponsiveness. Our findings demonstrate that the transition to unconsciousness is not linear, but rather consists of an interplay between transient brain networks reflecting different degrees of sleep depth.
\end{abstract}

Keywords Drowsiness $\cdot$ Responsiveness $\cdot$ EEG microstates $\cdot$ Brain connectivity

Handling Editor: Gregor Thut.

Electronic supplementary material The online version of this article (https://doi.org/10.1007/s10548-018-0689-9) contains supplementary material, which is available to authorized users.

Srivas Chennu

sc785@kent.ac.uk

1 Department of Clinical Neurosciences, University of Cambridge, Cambridge, UK

2 Department of Psychology, University of Cambridge, Cambridge, UK

3 School of Computing, University of Kent, Medway Building, Chatham Maritime ME4 4AG, UK

\section{Introduction}

As we fall asleep, our brain traverses a series of changes which accompany the loss of sensory awareness and responsiveness to the external world. Despite the subjective ability to classify retrospectively one's own state as "awake" or "asleep" (Hori et al. 1994), research continues to unravel the gradual transitions happening at behavioural (Ogilvie and Wilkinson 1984), cellular (Steriade et al. 1993), physiological (Prerau et al. 2014) and cognitive (Goupil and Bekinschtein 2012) level, starting with early drowsiness and continuing into the deep stages of sleep (Ogilvie 2001). Characterising these transitions and linking across physiological levels is an important step in the modern attempt to understand access-consciousness (Block 1996; Koch et al. 2016) and its fluctuations in natural, pathological and pharmacological alterations: sleep (Hobson and Pace-Schott 2002), disorders of consciousness (Giacino et al. 2014), sedation and anaesthesia (Alkire et al. 2008). 
The transition from wakefulness to sleep involves a progressive and sometimes nonlinear loss of responsiveness to external stimuli (Ogilvie and Wilkinson 1984). Behavioural unresponsiveness does not immediately imply unconsciousness (Overgaard and Overgaard 2011; Sanders et al. 2013). However, from the perspective of levels of consciousness (Laureys 2005), the capacity to respond to external stimuli offers an objective measurement in the process of transition between full wakefulness and sleep-induced unconsciousness. The question of how we stop responding to stimuli during drowsiness is related to, but distinct from an investigation of the stages of sleep conventionally defined by specific electrophysiological grapho-elements (Iber et al. 2007; Ogilvie 2001). Indeed, the loss of responsiveness is and distributed across sleep stages: one study found a rate of unresponsiveness of $28 \%$ in stage $1,76 \%$ in stage 2, and $95 \%$ in stage 3 of sleep (Ogilvie and Wilkinson 1984). Here, we are specifically interested in the neural markers that predict our inability to respond as we drift to sleep.

A traditional approach for investigating this question is to look at the changes in EEG spectral power and connectivity, which have been shown to vary across levels of consciousness. During relaxed wakefulness, the EEG of most human subjects is characterised by trains of alpha waves, at around $10 \mathrm{~Hz}$, originating from central-posterior cortical areas (Barry et al. 2007; De Gennaro et al. 2016; Niedermeyer 2005a). During the early onset of sleep, these alpha oscillations disappear and an alpha rhythm with a different cortical origin (Broughton and Hasan 1995) emerges in anterior regions (Tanaka et al. 1997), while theta power increases, particularly in central regions (Badia et al. 1994; Niedermeyer 2005b; Ogilvie 2001; Wright et al. 1995). Similarly, long-range alpha connectivity disintegrates at the onset of sleep, while lower-frequency theta and delta connectivity increases (Tanaka et al. 2000, 1998; Wright et al. 1995). Several power and connectivity patterns have been associated with the loss of consciousness, sometimes specifically with the loss of responsiveness, such as the anteriorisation of alpha power and connectivity in EEG, which has been described in drug-induced loss of responsiveness (Chennu et al. 2016a, b), and frontoparietal connectivity in fMRI, which has been proposed as a key signature of consciousness (Laureys 2005) and linked to external awareness (Vanhaudenhuyse et al. 2011). In EEG, the disruption of connectivity between frontal and parietal electrodes at alpha $(8-12 \mathrm{~Hz})$ frequencies has been shown to occur in disorders of consciousness (Chennu et al. 2014a, b) and sedation (Chennu et al. 2016a, b). Although it is still debated whether these are signatures of conscious processing or of processes that almost invariably accompany it (Farooqui and Manly 2017), brain connectivity patterns currently provide, in practice, useful insights into the transitions between levels of consciousness.
Another method that can be employed to investigate the rapidly changing global state of the brain is that of EEG microstates. A microstate represents a quasi-stable spatial topography of electric field on the scalp (Lehmann 1990, 1971; Lehmann et al. 1987). The conventional method of analysing microstates in a dataset involves running an unsupervised clustering algorithm on a set of EEG topographies of highest variance, followed by labelling of all EEG samples based on the similarity with the four obtained topographies (Murray et al. 2008; Pasqual-Marqui et al. 1995). Four consistent (Khanna et al. 2014) EEG microstate topographies have been identified in a large population of healthy subjects of all ages during resting-state wakefulness (Koenig et al. 2002a, b) and different microstates have been correlated with different cognitive modalities (Lehmann et al. 2010; Milz et al. 2015; Seitzman et al. 2016), but also with mental disorders, such as narcolepsy (Kuhn et al. 2015). A resting-state study of sleep (Brodbeck et al. 2012a, b) identified four EEG microstate topographies in all stages of sleep nearly identical to those of wakefulness, but occurring with altered temporal parameters. Notably, increased microstate duration was associated with deeper sleep. On the contrary, a different study (Cantero et al. 1999) reported a shorter duration of microstates and suggested a larger repertoire of brain states during the hypnagogic period. Microstates are thought to reflect momentary, global, synchronised (Koenig et al. 2005) networks of the brain, reflecting building blocks of large-scale cognitive processing required for the continuous stream of consciousness (Lehmann 1990). The neural sources underlying microstates are still being explored (Britz et al. 2010; Milz et al. 2017; Pascual-Marqui et al. 2014). Still, the dynamics of the sequence of microstates itself can be seen as a "syntax" of neural activity that is in and of itself an informative tool for modelling and understanding the rapidly-fluctuating global dynamics of the brain.

Brain connectivity and microstates hence provide complementary perspectives on the neurodynamics underlying the loss of responsiveness as we fall asleep. But what is the relationship between brain networks and microstates? There is evidence that transient brain networks can be resolved in electrophysiological data (Baker et al. 2014; Pascual-Marqui et al. 2014; Vidaurre et al. 2016), but it is an open question whether these networks co-occur with the lifetime of individual microstates. We investigate for the first time how spectral connectivity and EEG microstate dynamics interact as we lose responsiveness during drowsiness. We hypothesise that the spectral changes occurring with the loss of responsiveness mirror those observed in the transition to sleep (Ogilvie 2001), anaesthesia (Chennu et al. 2016a, b; Purdon et al. 2013) and in disorders of consciousness (Chennu et al. 2014a, b): namely, the disintegration of alpha networks, the loss of posterior alpha power, and the emergence of lower-frequency connectivity 
and power. Alongside, building on previous research on EEG microstate dynamics during sleep (Brodbeck et al. 2012a, b), we hypothesise similar changes in microstate dynamics accompanying the loss of responsiveness during drowsiness. Finally, given that resting-state network activity is known to fluctuate at millisecond level, we hypothesise that the neural changes in that occur during drowsiness underlie the dynamics of both brain networks and the microstates sequence. Specifically, we investigate the possibility that individual microstates co-occur with distinct transient brain networks, reflecting fleeting changes in the global state of the brain during drowsiness.

To address these questions, we use a subset of data from a previously reported auditory discrimination task where subjects became drowsy and unresponsive (Kouider et al. 2014). The task involved pressing a button corresponding to the classification of the auditory stimulus into one of two categories (object or animal). We obtain $5 \mathrm{~min}$ of data as subjects performed this task, before and after the loss of responsiveness due to drowsiness. We first characterise the responsive and unresponsive periods by analysing microstate-blind spectral power and connectivity changes in our dataset. Next, we describe the temporal parameters of EEG microstates during responsiveness and unresponsiveness. To test whether these parameters can reliably predict responsiveness to individual stimuli, we apply machine learning to predict responses and misses to stimuli in our task, based only on pre-stimulus microstate parameters. Finally, we investigate the brain connectivity underlying each of the four canonical microstates after the loss of responsiveness and highlight a previously unknown relationship between spectral connectivity and EEG microstates.

\section{Methods}

\section{Subjects}

Sixteen healthy, native English-speaking, right-handed young adults (mean age $=24, \mathrm{SD}=2.75 ; 6$ females) were selected for this experiment out of the 18 subjects from Experiment 1 in a previous study (Kouider et al. 2014). Two subjects from this dataset were excluded by visual inspection due to a failure to remain asleep for a period longer than $5 \mathrm{~min}$, as assessed using responsiveness to stimuli. The participants were directed to not consume stimulants like coffee and to sleep $1-2 \mathrm{~h}$ less than normally before the experiment. All of the subjects were assessed as easy sleepers on the Epworth Sleepiness Scale (scores 7-14). The participants signed a consent form and were reimbursed for their participation. The experiment was approved by the Cambridge Psychology Research Ethics Committee.

\section{Experimental Procedure}

The stimuli consisted of 96 spoken English words chosen from the CELEX lexical database (Linguistic Data Consortium, University of Pennsylvania). Half of the words denoted animals and the other half denoted objects. The subjects were asked to classify each stimulus in its respective category (animal or object) by pressing a button. The stimuli were presented through headphones, with an average distance of $8.4 \mathrm{~s}$ (minimum $6.2 \mathrm{~s}$ ) between consecutive stimuli, as the subjects were lying with their eyes closed in a reclining chair. To facilitate drowsiness, the task was performed in a dark, acoustically and electrically shielded EEG room, and the participants were told that they could fall asleep at any point during the experiment, although they were asked not to stop responding deliberately while still awake.

\section{EEG Data Acquisition}

The electroencephalogram was continuously recorded at 500 samples per second from $64 \mathrm{Ag} / \mathrm{AgCl}$ electrodes (NeuroScan Labs system) positioned and labelled according to the extended 10/20 system, with $\mathrm{Cz}$ as a reference and including vertical and horizontal electrooculography channels.

\section{EEG Pre-processing}

All analyses that follow were performed using custom MATLAB scripts (The MathWorks, Inc., Natick, Massachusetts, US). The EEGLAB toolbox (Delorme and Makeig 2004) was used to facilitate data pre-processing.

The data was filtered between 1 and $40 \mathrm{~Hz}$ and the full channel mean was subtracted from each channel for baseline correction. The HEOG and VEOG channels were removed. An Independent Component Analysis (ICA) decomposition was performed using the infomax ICA algorithm (Bell and Sejnowski 1995). Components capturing ocular or singlechannel artefacts were removed from the data by visual inspection and considering the correlation with the HEOG and VEOG channels. An average of $11.6(\mathrm{SD}=8.6)$ out of 63 components were removed per subject. Channel FT8 was interpolated using spherical interpolation in all subjects as it was noisy in most recordings. Finally, channels were rereferenced offline to the common average.

\section{Data Segmentation}

We classified responsive and unresponsive periods by inspecting the sequence of hits and misses to individual stimuli. We used a liberal window of $6 \mathrm{~s}$ to allow for a response to a stimulus, regardless of its correctness. A lack of response within $6 \mathrm{~s}$ was marked as a miss. The choice of a 6-s window for responsiveness was based on our own 
pilot studies, where we investigated the longest interval that subjects would make a response during drowsiness in a go task. However, note that most reaction times were below $3 \mathrm{~s}$ (Fig. 1) and the reaction times increased gradually and later in the task, indicating an increase in drowsiness. This was also established in a previous study on the same data (Kouider et al. 2014).

For balance across participants and the two behavioural states, a total of $5 \mathrm{~min}$ of responsiveness and $5 \mathrm{~min}$ of unresponsiveness were extracted from each recording $(150,000$ samples per state, per recording), as shown in Fig. 1. The responsiveness period was taken as the first $0.5-5.5 \mathrm{~min}$ of

\section{Reaction times (s) and data selection}

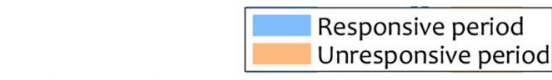

2

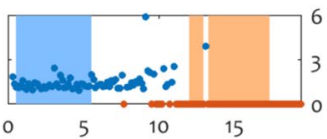

3

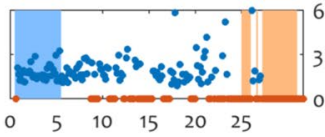

4

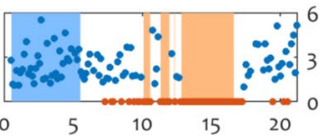

5

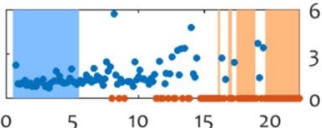

6

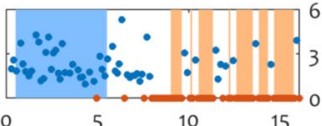

7

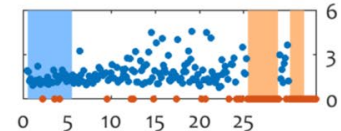

8

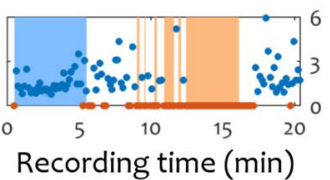

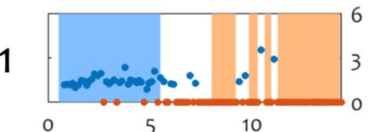

9

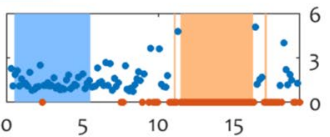

10

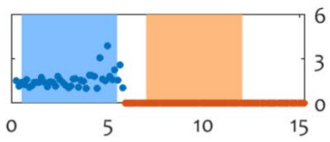

11

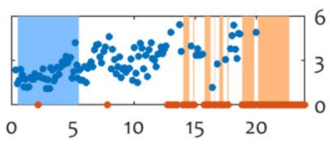

12

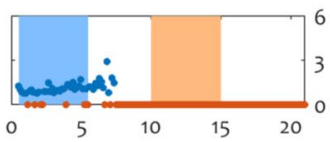

13

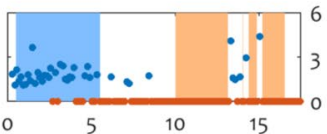

14

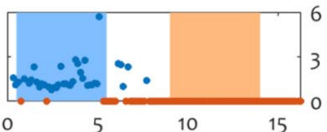

15

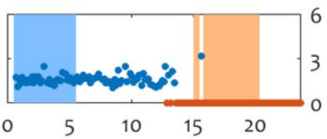

16

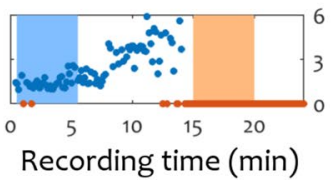

Fig. 1 Reaction times and data segmentation into responsiveness and unresponsiveness for individual participants. The horizontal axis represents recording time and the vertical axis represents reaction time in seconds. Blue markers indicate responses, while orange markers indicate misses. The blue area corresponds to the 5-min period of responsiveness, while the orange area corresponds to the 5-min period of unresponsiveness data in each recording, acquired immediately after the experiment began and the participants were still alert and wakeful. This was confirmed by checking that the large majority of the stimuli were followed by responses during this period; a very small number of occasional misses occurred in more than half of the participants during this period (e.g., due to unfamiliarity with the task), but they were not contiguous. Then, a period of unresponsiveness was selected by visual inspection of the hits and misses after the end of the responsiveness period, with the aim to find a 5-min interval consisting of as many misses as possible. If a response was present during the period labelled as unresponsiveness, the $10 \mathrm{~s}$ preceding and following the corresponding stimulus were excluded.

\section{Microstate Topographies}

The idea of electric microstates of the brain comes from the observation that the topography of the electric field recorded by EEG over the scalp does not fluctuate randomly, but is instead comprised of short periods of stability (Lehmann 1971). Four canonical microstates (Koenig et al. 2002a, b), conventionally labelled A, B, C and D, have been shown to be consistent across recording sessions (Khanna et al. 2014) and have been repeatedly confirmed in a wide range of health conditions and cognitive tasks across multiple studies (Britz et al. 2010; Brodbeck et al. 2012a, b; Grieder et al. 2016; Katayama et al. 2007; Kikuchi et al. 2011; Koenig et al. 1999; Kuhn et al. 2015; Milz et al. 2015; Nishida et al. 2013; Pascual-Marqui et al. 2014; Schlegel et al. 2012; Strelets et al. 2003; Tomescu et al. 2014a, b; Van de Ville et al. 2010).

To compute the microstate topographies, the Global Field Power (GFP), representing the standard deviation of the electrode values (Lehmann and Skrandies 1980), was first computed at each time point. As the number of GFP peaks varied across subjects and condition, we rounded down the minimum number of peaks available and retained the first 5000 peaks in each condition (responsiveness and unresponsiveness) from each recording.

The clustering algorithm was implemented in MATLAB and is presented in Box 1. The algorithm is based on a variant of the method first introduced by (Lehmann et al. 1987), as described in (Murray et al. 2008), and involves an unsupervised clustering of EEG samples into the specified number of classes that best explain the input samples. Note that topographical similarity is computed using the absolute value of the spatial correlation, and the polarity of the map is ignored, as topographies with inverted polarities are considered to be produced by the same neural generators (Michel et al. 2009). The maximum number of iterations was set to 1000 and the GEV delta was set to 1e-9. 
Box 1 Microstate clustering algorithm

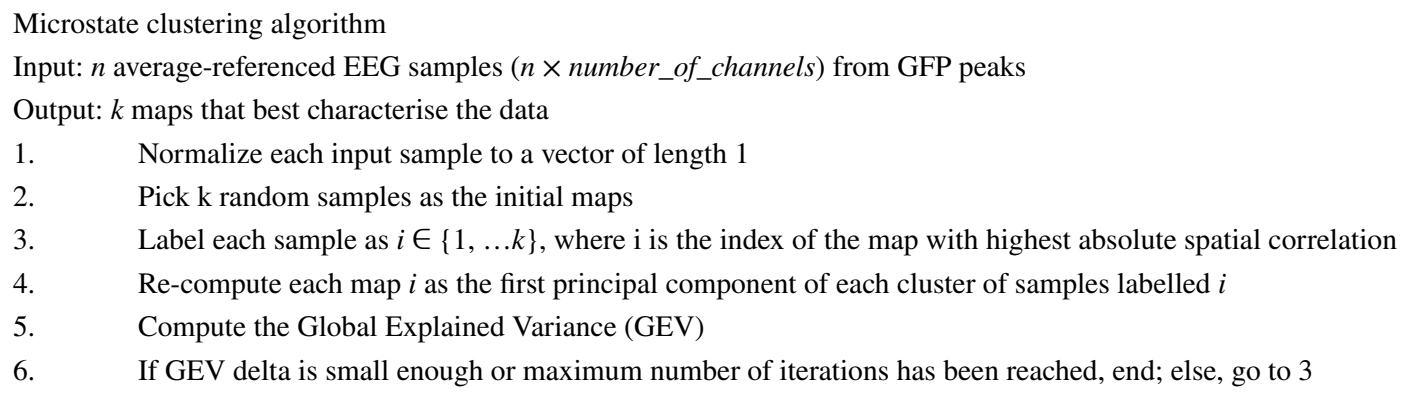

We initially employed a cross-validation criterion (Pasqual-Marqui et al. 1995) to determine the optimal number of microstates fitting the data, as performed in several previous studies (Brodbeck et al. 2012a, b; Koenig et al. 1999). However, we found that the cross-validation criterion produced different results for when the number of electrodes was down-sampled from 63 to 30 (7 and 4 maps, respectively). This sensitivity of the cross-validation criterion to the number of electrodes has been documented in previous literature (Murray et al. 2008). Hence, we decided to fix the number of microstates to four, in line with previous studies that also fix this number a priori (Khanna et al. 2014; Kikuchi et al. 2007; Koenig et al. 2002a, b; Milz et al. 2015; Schlegel et al. 2012; Strelets et al. 2003; Tomescu et al. 2014a, b).

\section{Microstate Labelling}

To obtain the sequence of EEG microstates characterising a recording, each EEG sample was individually assigned to the microstate with the highest corresponding spatial correlation. To correct for noisy assignments during polarity reversals (Koenig and Brandeis 2016), we applied a previouslydescribed temporal smoothing algorithm for the microstate sequence (Pasqual-Marqui et al. 1995) with parameter $b$ set to 5, corresponding to a smoothing neighbourhood of $20 \mathrm{~ms}$. This parameter was chosen to be in the range of mean microstate durations found by (Gärtner et al. 2015) using a model of microstate transition processes based on Markov chains (10 ms during wake, $34 \mathrm{~ms}$ during deep sleep).

\section{Microstate Properties}

Following the full labelling of each recording, three properties were computed for each microstate per state (responsiveness and unresponsiveness) and per recording:

- The microstate temporal coverage, also called the fractional occupancy, indicating the percentage of time spent in one microstate;
- The microstate duration, indicating the average length of continuous sequences labelled as one microstate;

- The Global Explained Variance (GEV), representing the amount of spatial correlation of the samples with their corresponding microstate topography, normalised by the GFP of the microstate topography.

\section{Statistics}

Interactions between microstate parameters and behavioural state (responsiveness and unresponsiveness) were performed using a two-way repeated measures ANOVA (Hogg and Ledolter 1987) with the microstate label and the behavioural state as factors. Sphericity was tested using Mauchly's test of sphericity (Mauchly 1940) and, where violated, was corrected using the Greenhouse-Geisser procedure (Greenhouse and Geisser 1959). The Tukey-Kramer method (Tukey 1949) was used to correct for multiple comparisons. After correction, a conventional threshold of $\mathrm{p}=0.05$ was used to assess significance. Unless otherwise specified, similar statistical tests were also performed for the measures that follow.

\section{Responsiveness Prediction}

We applied machine learning classification to explore whether microstate properties identified in the ongoing brain dynamics immediately preceding each auditory stimulus in the experimental trials could predict the presence or absence of a response to that stimulus. Importantly, all trials were considered for classification, both within and outside the periods labelled as responsive or unresponsive for the above microstate analysis.

Five seconds of EEG data immediately preceding a stimulus were used to generate the features for classification. We also investigated using shorter pre-stimulus time periods, down to $1 \mathrm{~s}$ of pre-stimulus data, but we found that classification accuracy increased with a larger amount of pre-stimulus data over which microstate dynamics could be more accurately estimated. At the same time, the amount of 
pre-stimulus data was restricted by the overlap with the previous trial. Trials overlapping with a response corresponding to the previous stimulus were excluded. By setting the pre-stimulus window to $5 \mathrm{~s}$, less than $10 \%$ of the trials were rejected due to overlap with the previous trial.

The input features generated for classification consisted of either individual microstate parameters computed during the 5-s pre-stimulus period in each trial, or a combination of these parameters. The parameters were those we previously characterised at the group level: namely the mean duration, mean coverage, and mean GEV for each microstate separately. The classifier was trained separately with the above individual and combined features. As a baseline, the theta-alpha ratio was also computed for each trial as the ratio between the total power spectral density at 5-6 and 9.5-10.5 Hz respectively, and used as an input feature for the classifier. The classification label for each trial was generated by labelling it as either as a timely response (1) or a miss (0).

We employed leave-one-subject-out cross-validation to test for the generalisability of the classifier's performance. For this, the data was split into 16 folds, with one fold corresponding to a single participant's trials. A support vector machine (SVM) (Christianini and Shawe-Taylor 2000) with a radial basis function kernel (Vert et al. 2004) was trained repeatedly by excluding one fold at the time from the training set and using it as a test set. The SVM was optimised by exhaustive search to use the optimal value for two parameters: the box constraint, which restricts the number of support vectors, and the kernel scale, both in the range [0.001, 1000] in logarithmic steps of 10.

Platt's method (Platt 1999) was used to generate class affiliation probabilities from the trained classifier. These continuously varying probabilities were then used to discriminate between responses and misses using both the Receiver Operator Characteristic (ROC) area under the curve (AUC) (Davis and Goadrich 2006) and the classification accuracy as the percentage of correct predictions out of the total number of predictions. The classification accuracy was also computed by setting the class discrimination threshold as the optimal operating point of the ROC curve and calculating the percentage of correct predictions, using the threshold as a boundary between the two target classes. We used Wilcoxon signed rank tests (Gibbons and Chakraborti 2011) to probe for significant differences between classification performances.

\section{Spectral Power and Connectivity Analyses}

Spectral power and connectivity during responsiveness and unresponsiveness was investigated in both microstate-blind and microstate-wise analyses. Before microstate-wise segmentation, the power spectral density was computed at each
EEG sample between 1 and $20 \mathrm{~Hz}$ as the absolute value of the Hilbert transform (Marple 1999) of the bandpass filtered data within windows of $0.25 \mathrm{~Hz}$. We performed most of the analysis on 1-20 Hz and focused on theta and alpha power, whose ratio has been shown to track the onset of sleep (Šušmáková and Krakovská 2007) and has been employed in other studies of drowsiness (Bareham et al. 2014) or impaired consciousness (Lechinger et al. 2013). For each channel in each recording, the spectral power at each frequency bin was divided by the sum of spectral power at all bins within $1-20 \mathrm{~Hz}$. This ratio was then multiplied by 100 , thereby obtaining relative power contribution percentage at that bin.

The connectivity within each pair of channels was analysed using the Weighted Phase Lag Index (WPLI) (Vinck et al. 2011), a connectivity measure based on the distribution of phase differences between signals designed to correct for volume conduction, which has been previously used to investigate brain connectivity during loss of consciousness (Chennu et al. 2016a, b, 2014a, b; Lee et al. 2013). The WPLI was obtained by pooling over the Hilbert phase of each sample labelled as belonging to a particular microstate (see Suppl. Figure 1).

For both spectral power and connectivity, the median across channels was computed to obtain one value per microstate and frequency of interest.

To further assess topographical changes in connectivity, two sets representing anterior $(\mathrm{AFz}, \mathrm{Fz}, \mathrm{FCz}, \mathrm{AF} 7, \mathrm{AF}$, F1, FC1, F3, FC3, F5, F7, AF8, AF4, F2, FC2, F4, FC4, F6, F8) and posterior (CPz, Pz, POz, Oz, P1, P2, PO3, PO4, O1, O2, P3, P5, P7, P4, P6, P8, CP3, CP1, CP2, CP4) electrodes were selected for analysis. Median WPLI connectivity was computed within the anterior and posterior groups separately for each participant.

\section{Results}

\section{Behavioural Data}

The distribution of responsiveness and reaction times over time confirmed that all the subjects were responsive for a minimum of $6 \mathrm{~min}$ in the beginning of the experimental session and became unresponsive at a later point. During the unresponsiveness period, participants predominantly reached sleep stage $\mathrm{N} 1$, and rarely $\mathrm{N} 2$, as detailed in (Kouider et al. 2014). Figure 1 shows the response reaction times and the misses in each participant, in addition to the selection of data for the subsequent microstate analysis. During responsive periods, most subjects had no more than one miss, with a mean of $2.125 \%$ of all responses during this period being misses. The grand average of reaction times during the responsive period was $1.5 \mathrm{~s}(\mathrm{SD}=0.7)$. 


\section{Spectral Power and Connectivity Dynamics}

Before delving into microstate analyses, we characterised the spectral power and connectivity patterns during responsive and unresponsive periods. We performed a microstate-blind analysis focusing on previously reported changes related to early sleep, but also anaesthesia and disorders of consciousness, including the alteration of posterior, frontal and frontoparietal connectivity within and between frontal and parietal electrodes. We focused on alpha and theta frequencies, as the theta-alpha ratio has been shown to be the best discriminator between wake and sleep stage 1 (Šušmáková and Krakovská 2007). We confirmed that there were no significant differences in the means of power and median connectivity in beta $(12-30 \mathrm{~Hz})$ or gamma $(30-40 \mathrm{~Hz})$ between the responsive and unresponsive periods. Based on the peaks present in alpha and theta bands in our data at 5.5 and $10 \mathrm{~Hz}$ (also see Fig. 6 later), we defined the spectral frequencies of interest in alpha range at $9.5-10.5 \mathrm{~Hz}$ and the theta frequencies of interest at 5-6 Hz, for both power contributions and connectivity.

We observed a decrease in mean alpha power contribution $(\mathrm{t}(1,15)=3.34, \mathrm{p}=0.0044$, Cohen's $\mathrm{d}=0.83)$ and an increase in mean theta power contribution $(\mathrm{t}(1,15)=7.1$, $\mathrm{p}=3.5 \mathrm{e}^{-6}$, Cohen's $\mathrm{d}=1.77$ ) going from responsiveness to unresponsiveness. As shown in Suppl. Figure 2, we noted an alpha peak in spectral power present around $10 \mathrm{~Hz}$ in the large majority of the participants during the responsive period, which faded during the unresponsive period. Lowerfrequency power in the theta frequency range increased during unresponsiveness. A single notable exception was Subject 12, whose alpha peak did not shift into theta range during the unresponsive period, however this subject was preserved in the analysis since there was no evidence that

\section{A Spectral power contribution}

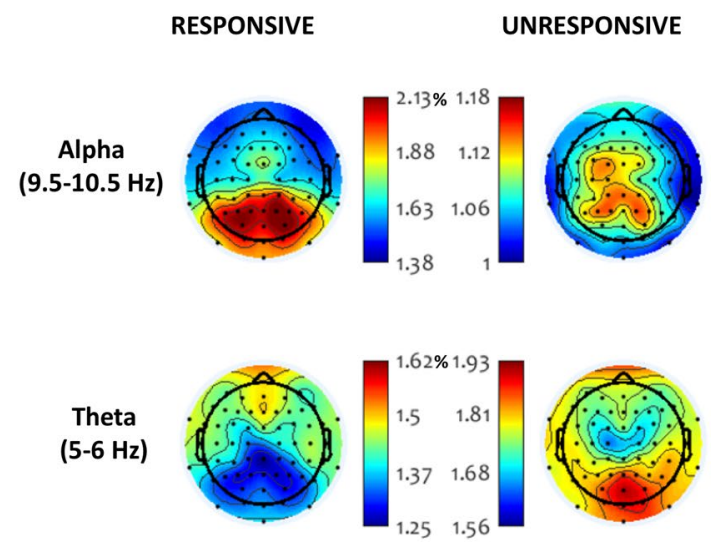

Fig. 2 Spectral power topography and WPLI frontoparietal connectivity at alpha $(9.5-10.5 \mathrm{~Hz})$ and theta $(5-6 \mathrm{~Hz})$ peaks before and after the loss of responsiveness. Values are averaged across partici- the experiment instructions were not followed. A grand average topographic plot of power at alpha and theta frequencies (Fig. 2a) revealed that the highest alpha power was located in the posterior area during responsiveness. During unresponsiveness, theta power was highest in posterior channels.

Investigating connectivity in alpha and theta frequencies using the WPLI, we observed the disintegration of longrange alpha band connections between frontal and parietal electrodes going from responsiveness to unresponsiveness (Fig. 2b and Suppl. Figure 3). A paired t-test confirmed that the median alpha connectivity between the anterior and posterior channels was significantly higher during responsiveness $(t(1,15)=3.4, p=0.003$, Cohen's $d=0.85)$. At the same time, an overall increase in median frontoparietal connectivity was observed in theta frequencies in unresponsiveness, but this was not significant $(t(1,15)=0.4, p=0.69$, Cohen's $\mathrm{d}=0.1)$.

\section{Microstate Topographies}

We applied the microstate clustering algorithm on the set of combined samples from the responsive and unresponsive periods from each subject, in order to obtain four microstate topographies. The resulting maps matched the four canonical microstate topographies commonly described in literature, denoted by letters A to D (Fig. 3). A breakdown of microstate topographies obtained for individual participants is also shown in Suppl. Figure 4.

\section{Microstate Parameters}

We investigated whether the dynamics of the rapid succession of microstates in the EEG remains the same before and after the loss of responsiveness. We computed the duration,

\section{B Frontoparietal WPLI connectivity}

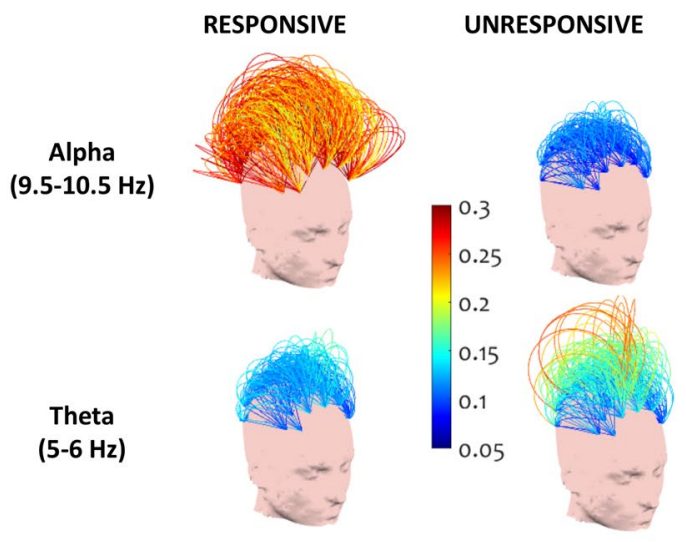

pants. With loss of responsiveness, power over parietal sensors and connectivity between frontal and parietal sensors shifted from the alpha to the theta band 


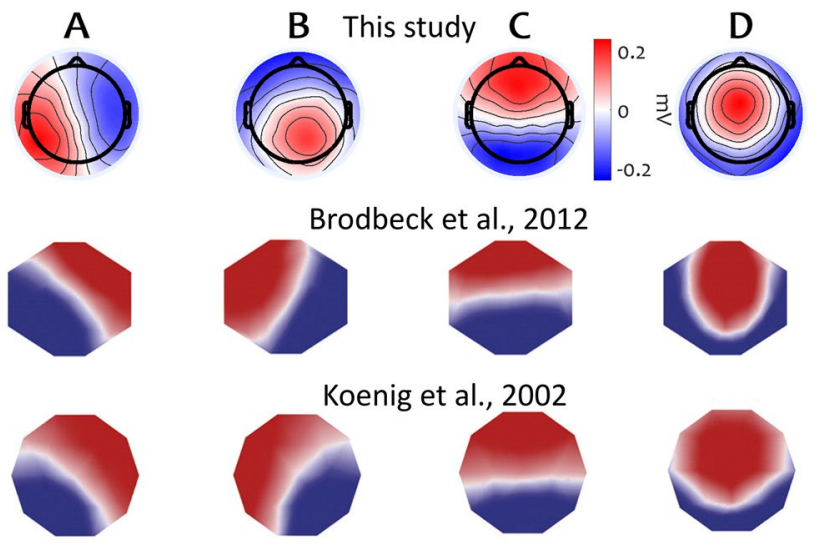

Fig. 3 Microstate topographies computed across all subjects. These topographies are plotted in correspondence with the four canonical microstate topographies commonly described in literature. Microstate topographies reported by Brodbeck et al. (2012a, b) and Koenig et al. $(2002 a, b)$ are shown for comparison (reproduced here with permission)

the temporal coverage and the global explained variance (GEV) of each microstate during responsiveness and during unresponsiveness (Fig. 4).

A repeated measures ANOVA with the microstate and the behavioural state (responsiveness and unresponsiveness) as factors found significant interactions between microstate and behavioural state in all of the three microstate parameters investigated: duration $\left(F_{\text {interaction }}=16.73\right.$, $P_{\text {interaction }}=2 \mathrm{e}^{-7}$, Cohen's $d=2.11$ ), temporal coverage $\left(F_{\text {interaction }}=13.08, P_{\text {interaction }}=3 \mathrm{e}^{-6}\right.$, Cohen's $\left.d=1.86\right)$ and $\operatorname{GEV}\left(F_{\text {interaction }}=17.95, P_{\text {interaction }}=8 \mathrm{e}^{-8}\right.$, Cohen's $\left.d=2.18\right)$. Further exploring the simple effect of state on the parameters within each microstate, the ANOVA revealed that the duration of all microstates was significantly increased during unresponsiveness $\left(P_{\text {state }, A}=0.0001, P_{\text {state } B}=0.003\right.$, $\left.P_{\text {state }, C}=0.0001, P_{\text {state } D}=3 \mathrm{e}^{-6}\right)$, in agreement with previous literature (Brodbeck et al. 2012a, b). Notably, microstate D had a striking increase in duration (Fig. 4a). At the same time, the temporal coverage of class D was significantly higher during unresponsiveness (Fig. 4b), whereas the coverage of microstate $\mathrm{B}$ was significantly lower during the same period $\left(P_{\text {state }, A}=0.056, P_{\text {state } B}=0.001, P_{\text {state }, C}=0.26\right.$, $P_{\text {state }, D}=1 \mathrm{e}^{-5}$ ). Similarly, the GEV of microstate D (Fig. 4c) was increased during unresponsiveness, while the GEV of microstates A and B were decreased $\left(P_{\text {state }, A}=0.0002\right.$, $\left.P_{\text {state }, B}=0.0002, P_{\text {state }, C}=0.17, P_{\text {state }, D}=2 \mathrm{e}^{-5}\right)$.

\section{Single-Trial Responsiveness Prediction}

We verified whether microstate parameters in the pre-stimulus window are able to dissociate responsiveness from unresponsiveness at an individual trial level during the full recordings, and whether these properties could be generalised across subjects.

Out of all trials, $8 \%$ contained a button press event during the $5 \mathrm{~s}$ preceding each stimulus and were excluded from further analysis. The remaining data had a balanced distribution of 1078 responses and 1117 misses out of a total of 2195 trials.

Training a radial basis function kernel support-vector machine repeatedly on the combined-microstate and microstate-wise features to predict the binary outcome of a trial, as a response or a miss, using one-subject-out cross-validation, confirmed that microstate dynamics were able to predict responsiveness at an individual trial level and across subjects, with a performance similar to that of the established theta-alpha ratio of spectral power (Fig. 5).

Combining the duration, temporal coverage, and GEV of each microstate to obtain a $4 \times 5$ input feature vector or each trial achieved a mean AUC of 0.8552 (mean classification accuracy of $75.2 \%$ ). In comparison, the theta-alpha ratio achieved a mean AUC of 0.8519 (mean classification accuracy of $74.24 \%$ ). A Wilcoxon signed rank test did not find significant differences between these performance distributions. When combined, the microstate features and the theta-alpha ratio obtained a mean AUC 0.8622 (mean classification accuracy of $77.1 \%$ ).

When used individually as input features for the classification, mean microstate duration performed remarkably well, achieving a mean AUC 0.8484 (mean classification accuracy of $76.1 \%$ ). According to Wilcoxon test, this was not significantly different from the classification performance of the combined microstate parameters. The duration of microstate $\mathrm{D}$ was significantly better at predicting responsiveness than microstates $A-C\left(p_{D-\{A, B, C}=\{0.0005,0.0006,0.002)\right.$.

It is worth noting that the one subject for whom the prediction performance was lower in the group was Subject 12 , who was also the only one whose alpha peak remained nearly unshifted after the loss of responsiveness (Suppl. Figure 2).

\section{Connectivity Differences Between Microstates}

Having established the characteristic temporal patterns exhibited by microstate sequences before and after drowsiness-induced loss of responsiveness, we next proceeded to investigate their relationship with the underlying spectral content of the EEG, and the modulation of this relationship as subjects become unresponsive. To this end, we investigated the power contributions and the WPLI connectivity computed across samples belonging to each microstate before and after the loss of responsiveness. While we do not assume a direct relation between neural sources of EEG microstates and EEG spectral power and connectivity, our aim is to assess whether the neural 

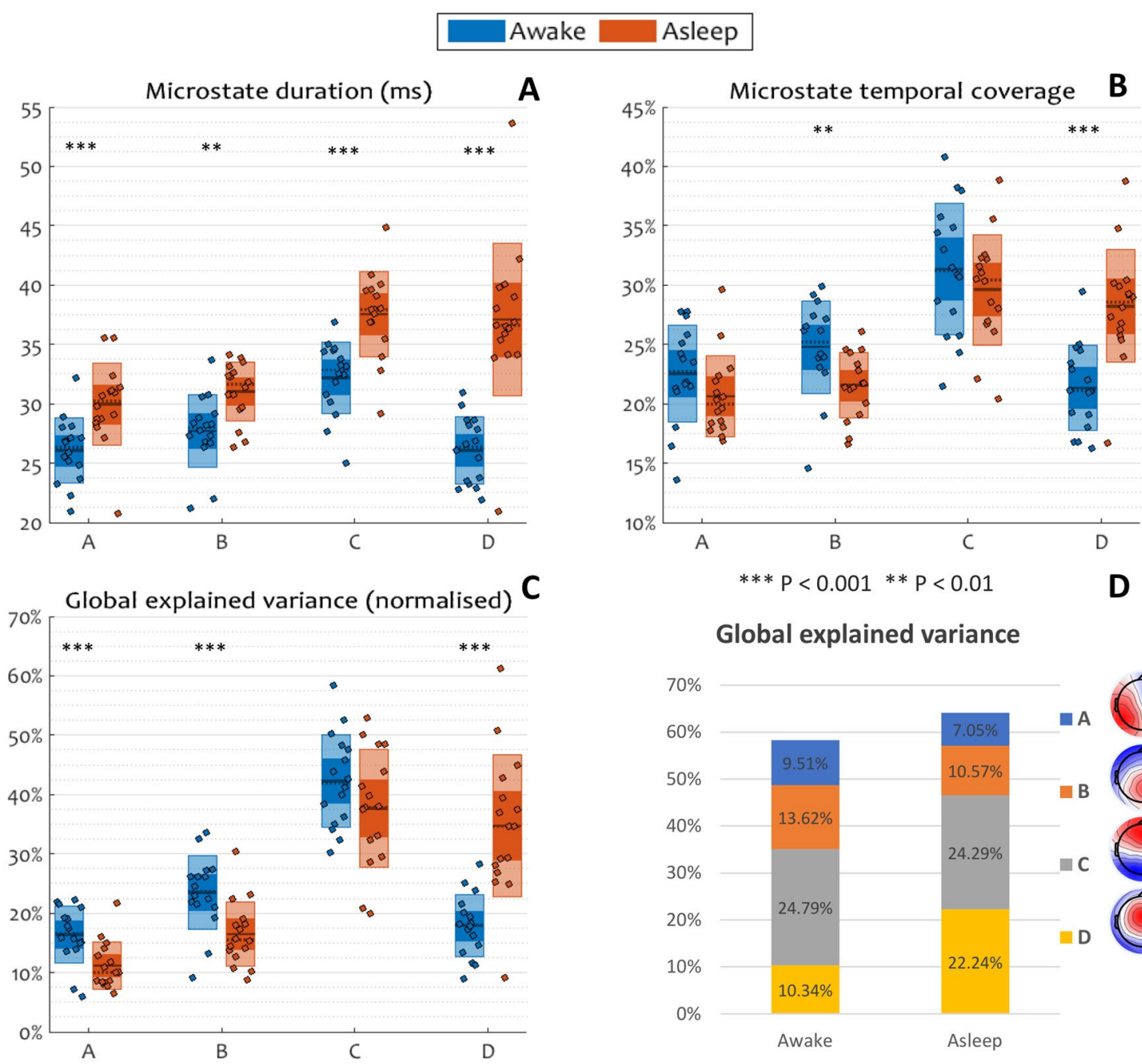

Global explained variance

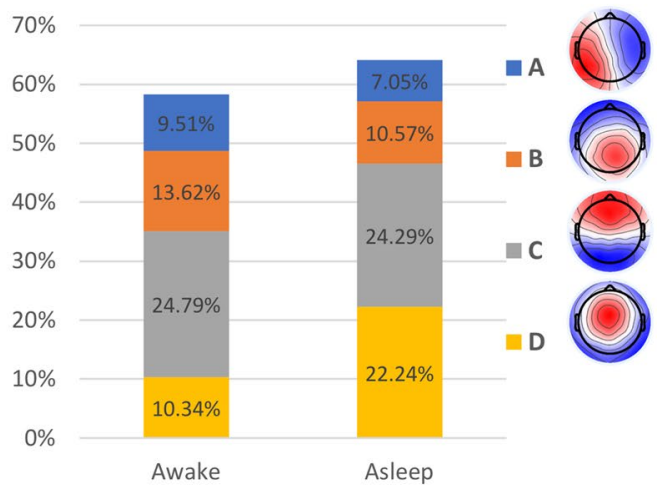

Fig. 4 Microstate parameters before and after the loss of responsiveness in drowsiness. Within each panel of grouped scatter box plots, inner boxes represent the standard error of the mean for each microstate parameter, and outer boxes represent the standard deviation. The mean is shown by a continuous line, the median is shown by a dotted

line, and individual participants are shown as dots. Asterisks show a significant within-subject main effect of state for a microstate. Duration, temporal coverage and GEV of microstate D all significantly increased during unresponsiveness

sources of microstates and sources of spectral measures covary at a fine temporal scale.

The spectral power contribution (Fig. 6a) displayed the characteristic alpha peak around $10 \mathrm{~Hz}$ during the responsive period, which faded during the unresponsive period into high power at low frequencies. This pattern was similar during all microstates.

Likewise, spectral connectivity (Fig. 6b) showed a peak at $10 \mathrm{~Hz}$ during responsiveness during all microstates, which faded during unresponsiveness. The only pattern dissociating between microstates during responsiveness was a decreased $10 \mathrm{~Hz}$ peak during microstate A. On the other hand, there was a noticeable difference in the level of connectivity during unresponsiveness between all

microstate periods, with microstates D and A exhibiting the highest and the lowest connectivity, respectively.

The effect size of the interaction between microstate and behavioural state (responsiveness and unresponsiveness) computed individually at each frequency was indeed generally higher in connectivity than in power (Fig. 6c). The effect size was largest in connectivity at 5.5 and $10 \mathrm{~Hz}$, corresponding to the theta and alpha peaks displayed during all microstates during the unresponsive and responsive periods, respectively. A peak in power contribution was also found at $13.5 \mathrm{~Hz}$, potentially due to the emergence of sleep spindles at the onset of sleep.

We also attempted to use pre-stimulus WPLI connectivity levels at alpha and theta frequencies in order to train a 


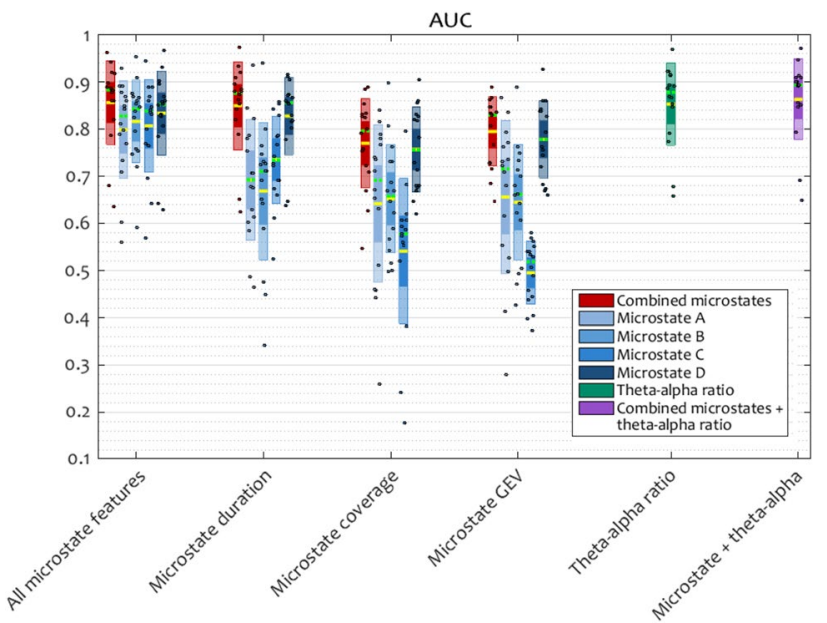

Fig. 5 Classification performance, computed as the area under the ROC curve, for a support-vector machine (SVM) trained using $5 \mathrm{~s}$ of pre-stimulus data to classify responses and misses. Input features to the classifier were microstate parameters or the theta-alpha ratio, individually or combined. Within each group of grouped scatter box plots, inner boxes represent the standard error of the mean, outer boxes represent the standard deviation. The mean is shown by a yellow line, the median is shown by a green line (where distinct from the mean), and individual participants are shown as dots. Microstate parameters were able to predict responsiveness at an individual trial level across subjects, with a performance similar to that of the thetaalpha ratio

classifier to predict responsiveness, using the same procedure as for the microstate spatiotemporal parameters. No classifiers could be obtained that exceeded a $60 \%$ mean accuracy, either microstate-wise or on the full set of prestimulus samples.

\section{Connectivity During Microstate D after the Loss of Responsiveness}

Gathering from the evidence of increased temporal presence of microstate D after the loss of responsiveness, as well as the higher connectivity displayed during this microstate during unresponsiveness in comparison with the microstates A-C, we next sought to understand the spectral connectivity patterns captured during microstate $\mathrm{D}$ in the selected alpha and theta ranges during the unresponsiveness period.

Previous literature suggests that anterior (frontal) and posterior (parietal) scalp regions of interest (ROI) show key changes in connectivity at the onset of sleep (Morikawa et al. 1997; Tanaka et al. 2000, 1998; Wright et al. 1995), during sedation and after brain injury (Chennu et al. 2017, 2014a, b, 2016). Building upon this, we investigated the withinanterior, within-posterior and between anterior-posterior connectivity during microstate $\mathrm{D}$ in comparison with microstates A-C. For this purpose, we performed three repeated measures ANOVA tests to compare the median connectivity during microstate $\mathrm{D}$ and that during each of the microstates A-C in each of the six conditions (two frequency bands $\mathrm{X}$ three scalp ROIs) during the unresponsive period. Within each condition, we corrected for the false discovery rate across the three tests (D vs. A, D vs. B and D vs. C) using Storey's procedure (Storey 2002).

Figure 7 exemplifies the most prominent differences we found in connectivity between samples covered by microstate $\mathrm{D}$ and microstates $\mathrm{A}-\mathrm{C}$ respectively, during unresponsiveness.

At the selected theta peak, the t-test results showed significantly higher median connectivity within the anterior region during microstate $\mathrm{D}$ compared to each of the other microstates $\left(\mathrm{P}_{\mathrm{D}-\{\mathrm{A}, \mathrm{B}, \mathrm{C}\}}=\{0.001,0.008\right.$, $0.001\}, \mathrm{t}_{\mathrm{D}-\{\mathrm{A}, \mathrm{B}, \mathrm{C}\}}=\{3.958,3.069,4.088\}$, Cohen's $\left.\mathrm{d}_{\mathrm{D}-\{\mathrm{A}, \mathrm{B}, \mathrm{C}\}}=\{0.990,0.767,1.022\}\right)$. Median connectivity between the anterior and posterior regions was also significantly higher during microstate $\mathrm{D}$ than in microstates $\mathrm{A}$ and $\mathrm{C}\left(\mathrm{P}_{\mathrm{D}-\{\mathrm{A}, \mathrm{B}, \mathrm{C}\}}=\{0.003,0.297,0.003\}, \mathrm{t}_{\mathrm{D}-\{\mathrm{A}, \mathrm{B}, \mathrm{C}\}}=\{3.578\right.$, $1.081,3.392\}$, Cohen's $\left.d_{D-\{A, B, C}=\{0.894,0.27,0.848\}\right)$. No significant differences were found in median connectivity within the posterior area.

Conversely, at the selected alpha peak, the repeated measures ANOVA showed significantly lower median connectivity within the posterior area during microstate $\mathrm{D}$ compared to microstates $\mathrm{A}-\mathrm{C}\left(\mathrm{P}_{\mathrm{D}\{\mathrm{A}, \mathrm{B}, \mathrm{C}\}}=\{0.033\right.$, $0.037,0.033\}, \mathrm{t}_{\mathrm{D}-\{\mathrm{A}, \mathrm{B}, \mathrm{C}\}}=\{2.686,2.294,2.559\}$, Cohen's $\left.d_{D-\{A, B, C\}}=\{0.672,0.573,0.67\}\right)$. At the same time, microstate $\mathrm{D}$ captured significantly higher within-anterior median connectivity than microstate $\mathrm{A}\left(\mathrm{P}_{\mathrm{D}-\{\mathrm{A}, \mathrm{B}, \mathrm{C}\}}=\{0.043\right.$, $0.617,0.055\}, \mathrm{t}_{\mathrm{D}-\{\mathrm{A}, \mathrm{B}, \mathrm{C}\}}=\{2.769,0.511,2.297\}$, Cohen's $\left.\mathrm{d}_{\mathrm{D}\{\mathrm{A}, \mathrm{B}, \mathrm{C}\}}=\{0.692,0.128,0.574\}\right)$. No significant difference in median connectivity between anterior and posterior regions was found during microstate $\mathrm{D}$ compared to microstates A-C.

These results confirmed that the timecourse of microstate D uniquely capture a simultaneous disintegration of posterior alpha connectivity and emergence of frontal theta connectivity, which is associated with the suppression of responsiveness at the onset of sleep.

\section{Discussion}

\section{Summary}

In this study, we used high-density EEG to explore the transient spatiotemporal and spectral dynamics of electrical brain activity before and after the loss of behavioural responsiveness due to drowsiness. Importantly, we examined the loss of responsiveness as participants became drowsy while performing a discrimination task. Hence by design, our study is in contrast to and complements studies 

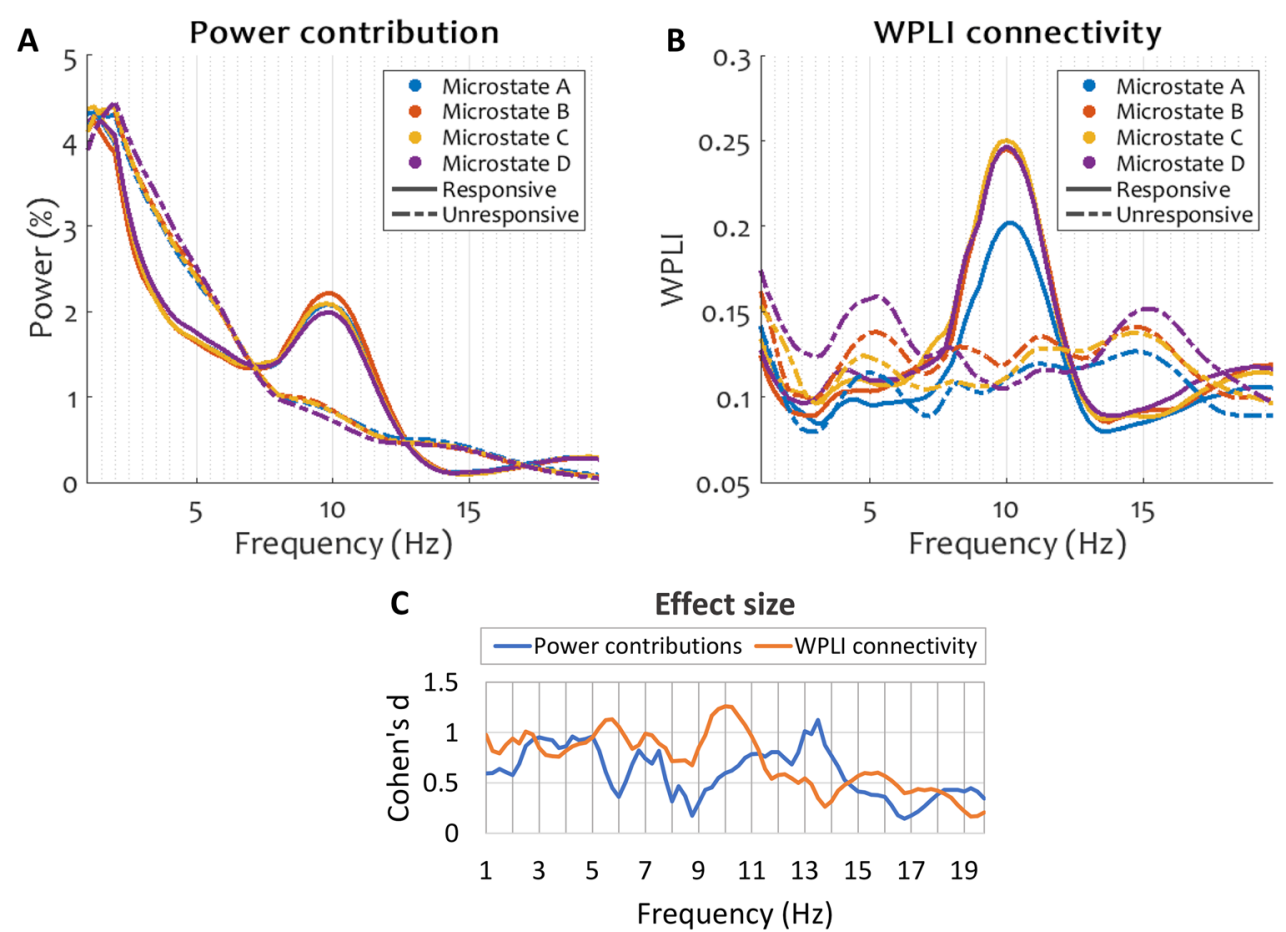

Fig. 6 Spectral power (a) and WPLI connectivity (b) captured during individual microstates before and after loss of responsiveness due to drowsiness. Channel-wise relative power at each frequency bin was calculated as the power at that bin as a percentage of total power within 1-20 Hz. Within each subject, for both relative power and WPLI connectivity, the median across channels is plotted. Fig- ures show the grand average over all subjects. c Shows the effect size, computed as Cohen's d, of the interaction between behavioural state and microstate at each frequency bin for power contributions and for connectivity. By convention, 0.2, 0.5 and 0.8 denote small, medium and large effect sizes, respectively. The interaction between microstate and behavioural state was stronger in connectivity than in power
Fig. 7 Frontal and frontoparietal WPLI connectivity at theta peak $(5-6 \mathrm{~Hz})$. Microstate D captured significantly higher theta connectivity within frontal and between frontoparietal sensors during unresponsiveness, compared to microstates $\mathrm{A}-\mathrm{C}$

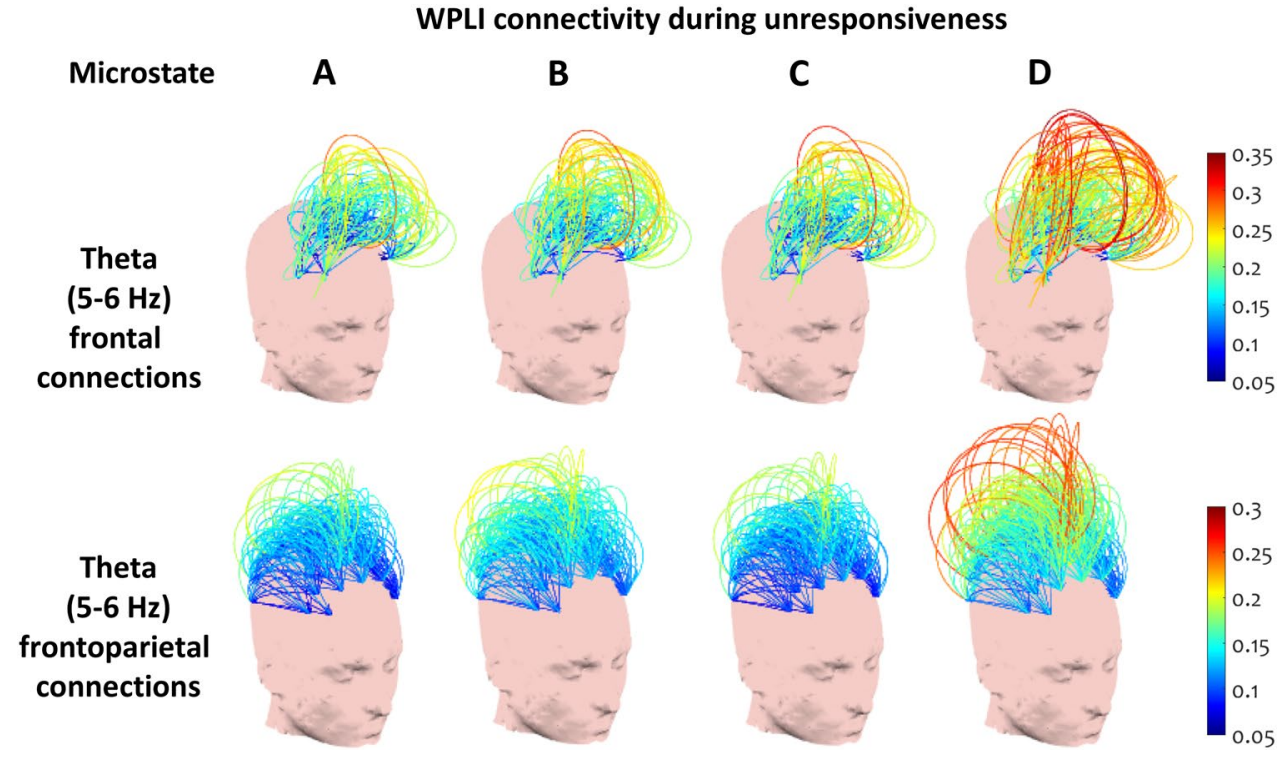

of resting brain activity in the absence of any task, which have often focused on an investigation of canonical sleep stages. Here, unresponsiveness - the failure to respond to the auditory cues elicited by increased drowsiness-provided an objective and non-invasive behavioural criterion in the transitional stage in between full wakefulness and early sleep.

To summarise our findings, we have shown that differences in spectral power and connectivity after the loss of responsiveness that have been previously shown to 
differentiate between healthy wakefulness and sleep, sedation and disorders of consciousness: a decrease in posterior alpha power and the emergence of theta power, as well as the disintegration of frontoparietal connectivity in alpha band. Further, microstate characteristics before and after the loss of responsiveness not only correlate with behaviour at the group level, but also predict behaviour at the level of individual experimental trials - when microstate D occurred more often during the pre-stimulus period, participants were less likely to generate a response to the subsequent stimulus. This relationship highlights a possible functional role of this microstate in modulating behaviour, and the predictive power of this signature to define the capacity to consciously respond to abstract/semantic stimuli. Finally, we discovered that while relative spectral power is similar across the temporal microstates, spectral connectivity is more distinctive. This non-uniform pattern of connectivity across microstates is modulated by the loss of responsiveness: the timecourse of microstate D captured significantly increased connectivity in the theta band after the loss of responsiveness, underpinning a novel profile of interaction between the temporal sequence of microstates and spectral brain connectivity.

\section{Alpha Power and Connectivity Characterise Responsive Wakefulness}

Our analysis of EEG connectivity before microstate segmentation strengthens the evidence for the fundamental role of the alpha networks in sustaining a state of responsive wakefulness. It is important to clarify that attribution of connectivity to specific neuroanatomy is limited by the scalp-level analysis we have conducted here, though previous research provides some pointers as to its neural origins. An independent study by Chennu et al. (2017) involving a different group of healthy adults and patients with brain injury has provided indirect information about the potential drivers of resting alpha connectivity, by correlating it with resting metabolism measured with PET imaging. As demonstrated in that study, the presence of a robust connectivity network in the alpha band was correlated with metabolic activity in frontal and parietal cortices encompassing both intrinsic and extrinsic awareness networks (Vanhaudenhuyse et al. 2010). Further, the body of literature based on simultaneous EEGfMRI recordings (Laufs et al. 2003a, b) has indicated negative correlations between alpha power and BOLD activity of frontoparietal areas known to be part of the attentional external awareness network. However, further research is needed to pinpoint the cortical and subcortical sources of the connectivity patterns and changes we have elucidated here.

Our analysis of scalp-level connectivity highlights that it is not the full disappearance of all connectivity that drives the loss of responsiveness, but specifically connectivity at alpha frequency. Indeed, literature shows that connectivity shifts from alpha into lower-frequency theta and delta frequencies in many contexts. This shift happens when consciousness fades (Chennu et al. 2014a, b, 2016; Ogilvie 2001; Tanaka et al. 2000, 1998; Wright et al. 1995), but also during natural fluctuations in alpha power during resting wakefulness, which accompany increases in theta power and BOLD activity in occipital and parietal areas (Laufs et al. 2006). In the larger picture of states and levels of consciousness, our findings confirm long-range alpha networks as a common marker of consciousness, whether this impairment is natural (sleep), pathological (disorders of consciousness) or pharmacological (sedation).

\section{Microstate D Predicts Responsiveness Across Subjects}

Upon examining the spatiotemporal parameters of the canonical EEG microstates, we found an increase in temporal coverage after the loss of responsiveness uniquely specific to microstate $\mathrm{D}$, along with an increase in its global explained variance, as compared to responsive periods. While the duration of all microstates was longer during unresponsiveness, the duration of microstate D had a prominent relative increase. In contrast, the temporal coverage of microstate B decreased in the unresponsive period, as did the global explained variance of microstates A and B. Further, we demonstrated that the general state of awareness, as reflected in the ongoing dynamics of pre-stimulus EEG microstates, are indeed informative of the capacity of a subject to respond to a stimulus during drowsiness at an individual trial level. This finding echoes similar evidence from the literature, where pre-stimulus microstate properties predict perception of weak stimuli (Britz et al. 2014), accuracy of working memory (Muthukrishnan et al. 2016) and perceptual shifts between bistable stimuli (Britz et al. 2009). Again, the special significance of microstate D during unresponsiveness was visible from its increased ability to predict the likelihood of a response, in comparison with microstates A-C. In addition, we showed that the increase in duration of this microstate is the best predictor of responsiveness among all the microstate parameters.

We note that the durations of the microstates we obtained were significantly lower than previous reports in the literature (Brodbeck et al. 2012a, b; Tomescu et al. 2014a, b, 2018). This might be due in part to our analytical methodology: as specified in the methods, we used a smoothing neighbourhood of $20 \mathrm{~ms}$, in keeping with the range of mean microstate durations reported in the re-analysis of data from (Brodbeck et al. 2012a, b) reported by Gärtner et al. (2015, Neuroimage). However, our smaller durations could also be attributed to the fact that our participants were performing an experimental task, which might have led to more rapid changes in dynamical brain states. This was in contrast to the 
reports above, in which data were collected in the absence of any task. Nevertheless, despite the shorter durations, we confirmed the expected presence of WPLI connectivity in the alpha band within each microstate during wakefulness. Speculatively, the finer temporal granularity of our microstate decomposition might have made the differences in connectivity between microstates more apparent during the transition to sleep.

Our usage of machine learning allows us to quantify the performance of the model using its discrimination accuracy, which speaks for the real-world applicability of the method (Breiman 2001). Moreover, one-subject-out cross-validation allows us to infer that these results are generalizable across people. At the same time, as expected, individual variability caps the maximum possible accuracy when predicting responsiveness. Our results suggest that this cap is around an accuracy of $75 \%$ (mean AUC around 0.85). Interestingly, the theta-alpha ratio, which we used as a baseline given its sensitivity as a sleep index (Šušmáková and Krakovská 2007), achieved a similar classification accuracy as the microstatebased input features. This suggests that microstate dynamics and spectral oscillations are potentially correlated. Intriguingly, we were not able to use connectivity as a feature to train a suitable classifier for responsiveness during drowsiness, either considering or ignoring the microstate sequence, despite strong evidence of major connectivity changes occurring before and after the loss of responsiveness. This suggests that connectivity better predicts the level of consciousness estimated over longer time scales, whereas spatiotemporal microstate dynamics capture short-term changes in brain state that predict responsiveness.

\section{Microstate D Captures a Distinct Connectivity Profile After Loss of Responsiveness}

Alongside the distinctive increase in temporal coverage and duration of microstate $\mathrm{D}$, we found a singular spectral connectivity pattern during this microstate after loss of responsiveness, indicating increased median connectivity in theta band, particularly in connections within frontal and between frontal and parietal electrodes. At the same time, median posterior connectivity during microstate $\mathrm{D}$ was reduced during unresponsiveness. Hence, the timecourse of microstate D appears to uniquely capture a connectivity pattern specific to deeper stages of sleep, in comparison with other microstates present during the same sleep stage. (Britz et al. 2010) correlated the microstate timecourses with the timecourse of average spectral power within canonical frequency bands. They reported finding no relationship between microstate dynamics and the spectral properties of the EEG signal. Our findings represent the first demonstration that in fact, spectral brain connectivity in fact presents a significant interaction with temporal microstate dynamics, underpinned by the connectivity captured by microstate D. Estimation of connectivity from EEG can be affected by volume conduction and referencing method. We have aimed to minimise the influence of the former with the use of WPLI-based connectivity. Further, we verified that re-analysis of WPLI connectivity with reference-free current source density estimates (Kayser and Tenke 2015) identified strong interactions in the alpha and theta bands, similar to those presented in Fig. 6b (see Suppl. Figure 5).

There currently exists no consensus on the meaning of individual microstates in terms of their neural generators. However, microstate D has occasionally been linked to attentional networks. In a study of fMRI resting-state networks, (Britz et al. 2010) showed a higher correlation of microstate D with ventral and dorsal frontoparietal networks, functionally associated with attention switching and directing attention towards external salient stimuli. A decreased duration of this microstate has been reported in schizophrenia (Koenig et al. 1999; Lehmann et al. 2005; Nishida et al. 2013; Tomescu et al. 2014a, b) and hallucination (Kindler et al. 2011) - two conditions involving impairments in task switching and attention (Collerton et al. 2005; Cornblatt and Keilp 1994). An investigation of modalities of thinking found an increased microstate $\mathrm{D}$ duration in restingstate compared to visual and verbal task periods (Milz et al. 2015); this was also interpreted as a confirmation of the previously-mentioned study by (Britz et al. 2010) due to a higher probability of attention switching during rest (high microstate D duration), as opposed to performing a single goal-oriented task (lower microstate $\mathrm{D}$ duration). On the other hand, (Seitzman et al. 2016) have found an increased duration of microstate $\mathrm{D}$ during a cognitive task as compared to wakeful rest.

Given the weak evidence in the literature associating microstate $\mathrm{D}$ with task-related attention networks, we are cautious in interpreting our findings on this basis. A previous study on the same data (Kouider et al. 2014) found that a correct response to stimuli is still prepared during unresponsiveness, suggesting preserved attention. It is possible that our findings indicate more demand from attention networks as drowsiness increases and subjects become unable to respond to the task. In study of microstates during sleep in the absence of any task, (Brodbeck et al. 2012a, b) did not observe an increase in this microstate during sleep. This suggests that microstate D might indeed be specifically related to the experimental task. Further, this interpretation is compatible with a study by Katayama et al. (2007), which found that the duration of microstate $\mathrm{D}$ was increased in light (but not deep) hypnosis, a state which produces similar EEG patterns to sleep-induced unresponsiveness (Barker and Burgwin 1949).

Nonetheless, the spatiotemporal and spectral connectivity dynamics observed in microstate $\mathrm{D}$ after the loss of 
responsiveness yield an important insight into the dynamics of the transition to sleep. While connectivity averaged during all microstates reflects typical changes commonly found in the loss of consciousness in the onset of sleep, anaesthesia or disorders of consciousness-weaker alpha and stronger theta long-range networks-the individual timecourse of microstate D captures significantly stronger patterns, despite having a duration no longer than $40 \mathrm{~ms}$. This suggests that, after the loss of responsiveness, the process of falling asleep is not necessarily linear, but rather consists of an interplay between distinct networks, captured by different microstates, which are at different points along the transition between wakeful and asleep modes of operation. It is worth noting that many subjects often became variable in their response times, and eventually unresponsive, within 5-7 min of starting the recording (see Fig. 1), highlighting the natural onset of drowsiness that could confound many experimental designs, if not appropriately controlled for (Noreika et al. 2017; Tagliazucchi and Laufs 2014). Further, our work might lend itself to explaining one of the current riddles of sleep research: why is it that, despite the establishment of a series of clear EEG markers delimiting wake and several stages of sleep, finding an EEG-based threshold to separate between the subjective intuition of being awake or asleep has not yet been achieved? Indeed, it has been reported by Hori et al. (1994) that $26 \%$ of all subjects stated that they had been awake at times when their EEG was classified as stage 2 sleep, which is commonly used to define "true sleep" (Ogilvie 2001). The rapid fluctuation of brain networks, some of which are closer to wakefulness (during microstates A-C) and others closer to sleep (during microstate $\mathrm{D}$ ) could be the reason why our momentary introspective state of being "awake" and "asleep" might not concur with a coarse-grained assessment of EEG over many seconds of data, as usually done during the identification of sleep stages. Instead, our findings here highlight that further research should focus on the rapidly changing dynamics of brain networks that appear to capture key dynamics relevant to our behavioural and perhaps even introspective state, as we drift into unconsciousness.

Acknowledgements We thank Louise Goupil for collecting the data for this experiment.

Open Access This article is distributed under the terms of the Creative Commons Attribution 4.0 International License (http://creativeco mmons.org/licenses/by/4.0/), which permits unrestricted use, distribution, and reproduction in any medium, provided you give appropriate credit to the original author(s) and the source, provide a link to the Creative Commons license, and indicate if changes were made.

\section{References}

Alkire MT, Hudetz AG, Tononi G (2008) Consciousness anesthesia Science 322:876-880

Badia P, Wright KPJ, Wauquier A (1994) Fluctuations in single-hertz EEG activity during the transition to sleep. In: Ogilvie RD, Harsh JR (eds) Sleep onset normal and abnormal processes. APA, Washington, pp 201-218

Bareham CA, Manly T, Pustovaya OV, Scott SK, Bekinschtein TA (2014) Losing the left side of the world: rightward shift in human spatial attention with sleep onset. Sci Rep 4:5092

Barker W, Burgwin S (1949) Brain wave patterns during hypnosis, hypnotic sleep and normal sleep. Arch Neurol Psychiatry $62: 412-420$

Baker AP, Brookes MJ, Rezek IA, Smith SM, Behrens T, Smith PJP, Woolrich M (2014) Fast transient networks in spontaneous human brain activity. Elife 2014:1-18

Barry RJ, Clarke AR, Johnstone SJ, Magee CA, Rushby JA (2007) EEG differences between eyes-closed and eyes-open resting conditions. Clin Neurophysiol 118:2765-2773

Bell A, Sejnowski TJ (1995) Fast blind separation based on information theory. In: Proceedings of the international symposium on nonlinear theory, pp 43-47

Block N (1996) How can we find the neural correlate of consciousness? Trends Neurosci 19:456-459

Britz J, Landis T, Michel CM (2009) Right parietal brain activity precedes perceptual alternation of bistable stimuli. Cereb Cortex 19:55-65. https://doi.org/10.1093/cercor/bhn056

Britz J, Van De Ville D, Michel CM (2010) BOLD correlates of EEG topography reveal rapid resting-state network dynamics. Neuroimage 52:1162-1170

Britz J, Diaz Hernandez L, Ro T, Michel CM (2014) EEG-microstate dependent emergence of perceptual awareness. Front Behav Neurosci 8:163. https://doi.org/10.3389/fnbeh.2014.00163

Brodbeck V et al (2012a) EEG microstates of wakefulness and NREM sleep. Neuroimage 62:2129-2139. https://doi.org/10.1016/j. neuroimage.2012.05.060

Brodbeck V, Kuhn A, von Wegner F, Morzelewski A, Tagliazucchi E, Borisov S, Michel CM, Laufs H (2012b) EEG microstates of wakefulness and NREM sleep. Neuroimage 62:2129-2139

Broughton R, Hasan J (1995) Quantitative topographic electroencephalographic mapping during drowsiness and sleep onset. J Clin Neurophysiol 12:372-386

Cantero JL, Atienza M, Salas RM, Gómez CM (1999) Brain spatial microstates of human spontaneous alpha activity in relaxed wakefulness, drowsiness period, and REM sleep. Brain Topogr $11: 257-263$

Chennu S et al (2014a) Spectral signatures of reorganised brain networks in disorders of consciousness. PLOS Comput Biol 10:e1003887. https://doi.org/10.1371/journal.pcbi.1003887

Chennu S, Finoia P, Kamau E, Allanson J, Williams GB, Monti MM, Noreika V, Arnatkeviciute A, Canales-Johnson A, Olivares F, Cabezas-Soto D, Menon DK, Pickard JD, Owen AM, Bekinschtein TA (2014b) Spectral signatures of reorganised brain networks in disorders of consciousness. PLoS Comput Biol 10:e1003887

Chennu S, O'Connor S, Adapa R, Menon DK, Bekinschtein TA (2016a) Brain connectivity dissociates responsiveness from drug exposure during propofol-induced transitions of consciousness. PLOS Comput Biol 12:e1004669. https://doi.org/10.1371/journ al.pcbi.1004669

Chennu S, O'Connor S, Adapa R, Menon DK, Bekinschtein TA (2016b) Brain connectivity dissociates responsiveness from drug exposure during propofol-induced transitions of consciousness. PLOS Comput Biol 12, e1004669 
Chennu S et al (2017) Brain networks predict metabolism, diagnosis and prognosis at the bedside in disorders of consciousness. Brain 140:2120-2132. https://doi.org/10.1093/brain/awx163

Christianini N, Shawe-Taylor J (2000) Support vector machines. Cambridge University Press, Cambridge

Collerton D, Perry E, McKeith I (2005) Why people see things that are not there: a novel perception and attention deficit model for recurrent complex visual hallucinations. Behav Brain Sci 28:737-757

Cornblatt BA, Keilp JG (1994) Impaired attention, genetics, and the pathophysiology of schizophrenia. Schizophr Bull 20:31-46

Davis J, Goadrich M (2006) The relationship between precision-recall and ROC curves. In: Proc. 23rd Int. Conf. Mach. Learn.ICML'06, pp. 233-240

De Gennaro L, Ferrara M, Curcio G, Cristiani R (2016) Antero-posterior EEG changes during the wakefulness and sleep transition. Clin Neurophysiol 112:1901-1911

Delorme A, Makeig S (2004) EEGLAB: an open source toolbox for analysis of single-trial EEG dynamics including independent component analysis. J Neurosci Methods 134:9-21

Farooqui AA, Manly T (2017) When attended and conscious perception deactivates fronto-parietal regions. Cortex 107:166-179

Gärtner M, Brodbeck V, Laufs H, Schneider G (2015) A stochastic model for EEG microstate sequence analysis. Neuroimage 104:199-208

Giacino JT, Fins JJ, Laureys S, Schiff ND (2014) Disorders of consciousness after acquired brain injury: the state of the science. Nat Rev Neurol 10:99-114

Gibbons JD, Chakraborti S (2011) Nonparametric statistical inference. Springer, New York

Goupil L, Bekinschtein TA (2012) Cognitive processing during the transition to sleep. Arch Ital Biol 150:140-154

Greenhouse SW, Geisser S (1959) On methods in the analysis of profile data. Psychometrika 24:95-112

Grieder M, Koenig T, Kinoshita T, Utsunomiya K, Wahlund LO, Dierks T, Nishida K (2016) Discovering EEG resting state alterations of semantic dementia. Clin Neurophysiol 127:2175-2181

Hobson JA, Pace-Schott EF (2002) The cognitive neuroscience of sleep: neuronal systems, consciousness and learning. Nat Rev Neurosci 3:679-693

Hogg RV, Ledolter J (1987) Engineering statistics. MacMillan, New York

Hori T, Hayashi M, Morikawa T (1994) Topographical EEG changes and the hypnagogic experience. In: Ogilvie RD, Harsh JR (eds) Sleep onset: normal and abnormal processes. American Psychological Association, Washington, pp 237-253

Iber C, Ancoli-Israel S, Chesson AL Jr, Quan SF (2007) The AASM manual for the scoring of sleep and associated events: rules terminology and technical specifications, 1st edn. American Academy of Sleep Medicine, Westchester

Katayama H, Gianotti LRR, Isotani T, Faber PL, Sasada K, Kinoshita T, Lehmann D (2007) Classes of multichannel EEG microstates in light and deep hypnotic conditions. Brain Topogr 20:7-14

Kayser J, Tenke CE (2015) On the benefits of using surface Laplacian (current source density) methodology in electrophysiology. Int J Psychophysiol 97:171-173. https://doi.org/10.1016/j.ijpsy cho.2015.06.001

Khanna A, Pascual-Leone A, Farzan F (2014) Reliability of restingstate microstate features in electroencephalography. PLoS ONE 9:e114163

Kikuchi M, Koenig T, Wada Y, Higashima M, Koshino Y, Strik W, Dierks T (2007) Native EEG and treatment effects in neuroleptic-naïve schizophrenic patients: time and frequency domain approaches. Schizophr Res 97:163-172
Kikuchi M, Koenig T, Munesue T, Hanaoka A, Strik W, Dierks T, Koshino Y, Minabe Y (2011) EEG microstate analysis in drugnaive patients with panic disorder. PLoS ONE 6:2-7

Kindler J, Hubl D, Strik WK, Dierks T, Koenig T (2011) Restingstate EEG in schizophrenia: auditory verbal hallucinations are related to shortening of specific microstates. Clin Neurophysiol 122:1179-1182

Koch C, Massimini M, Boly M, Tononi G (2016) Neural correlates of consciousness: progress and problems. Nat Rev Neurosci 17:307-321

Koenig T, Brandeis D (2016) Inappropriate assumptions about EEG state changes and their impact on the quantification of EEG state dynamics. Neuroimage 125:1104-1106

Koenig T, Lehmann D, Merlo MCG, Kochi K, Hell D, Koukkou M (1999) A deviant EEG brain microstate in acute, neurolepticnaive schizophrenics at rest. Eur Arch Psychiatry Clin Neurosci 249:205-211

Koenig T, Prichep L, Lehmann D, Sosa PV, Braeker E, Kleinlogel H, Isenhart R, John ER (2002a) Millisecond by millisecond, year by year: normative EEG microstates and developmental stages. Neuroimage 16:41-48

Koenig T et al (2002b) Millisecond by millisecond, year by year: normative EEG microstates and developmental stages. Neuroimage 16:41-48. https://doi.org/10.1006/nimg.2002.1070

Koenig T, Studer D, Hubl D, Melie L, Strik WK (2005) Brain connectivity at different time-scales measured with EEG. Philos Trans R Soc B Biol Sci 360:1015-1024

Kouider S, Andrillon T, Barbosa LS, Goupil L, Bekinschtein TA (2014) Inducing task-relevant responses to speech in the sleeping brain. Curr Biol 24:2208-2214

Kuhn A, Brodbeck V, Tagliazucchi E, Morzelewski A, von Wegner F, Laufs H (2015) Narcoleptic patients show fragmented EEG-microstructure during early NREM sleep. Brain Topogr 28:619-635

Laufs H, Kleinschmidt A, Beyerle A, Eger E, Salek-Haddadi A, Preibisch C, Krakow K (2003a) EEG-correlated fMRI of human alpha activity. NeuroImage 19:1463-1476

Laufs H, Krakow K, Sterzer P, Eger E, Beyerle A, Salek-Haddadi A, Kleinschmidt A (2003b) Electroencephalographic signatures of attentional and cognitive default modes in spontaneous brain activity fluctuations at rest. Proc Natl Acad Sci USA 100:1105311058. https://doi.org/10.1073/pnas.1831638100

Laufs H, Holt JL, Elfont R, Krams M, Paul JS, Krakow K, Kleinschmidt A (2006) Where the BOLD signal goes when alpha EEG leaves. NeuroImage 31:1408-1418

Laureys S (2005) The neural correlate of (un)awareness: lessons from the vegetative state. Trends Cogn Sci 9:556-559

Lechinger J, Bothe K, Pichler G, Michitsch G, Donis J, Klimesch W, Schabus M (2013) CRS-R score in disorders of consciousness is strongly related to spectral EEG at rest. J Neurol 260:2348-2356

Lee H, Mashour G, Noh G-J, Kim S, Lee U (2013) Reconfiguration of network hub structure after propofol-induced unconsciousness. Anesthesiology 119:1347-1359

Lehmann D (1971) Multichannel topography of human alpha EEG fields. Electroencephalogr Clin Neurophysiol 31:439-449

Lehmann D (1990) Brain electric microstates and cognition: the atoms of thought. In: John ER (ed) Machinery of the mind. Springer, New York, pp 209-224

Lehmann D, Skrandies W (1980) Reference-free identification of components of checkerboard-evoked multichannel potential fields. Electroencephalogr Clin Neurophysiol 48:609-621

Lehmann D, Ozaki H, Pal I (1987) EEG alpha map series: brain microstates by space-oriented adaptive segmentation. Electroencephalogr Clin Neurophysiol 67:271-288 
Lehmann D, Faber PL, Galderisi S, Herrmann WM, Kinoshita T, Koukkou M, Mucci A, Pascual-Marqui RD, Saito N, Wackermann J, Winterer G, Koenig T (2005) EEG microstate duration and syntax in acute, medication-naïve, first-episode schizophrenia: a multi-center study. Psychiatry Res Neuroimaging 138:141-156

Lehmann D, Pascual-Marqui RD, Strik WK, Koenig T (2010) Core networks for visual-concrete and abstract thought content: a brain electric microstate analysis. Neuroimage 49:1073-1079

Marple L (1999) Computing the discrete-time "analytic" signal via FFT. IEEE Trans Signal Process 47:2600-2603

Mauchly JW (1940) Significance test for sphericity of a normal n-variate distribution. Ann Math Stat 11:204-209

Michel CM, Koenig T, Brandeis D (2009) Electrical neuroimaging in the time domain. In: Michel CM, Koenig T, Brandeis D, Gianotti LRR, Wackermann J (eds) Electrical neuroimaging. Cambridge University Press, Cambridge, pp 111-144

Milz P, Faber PL, Lehmann D, Koenig T, Kochi K, Pascual-Marqui RD (2015) The functional significance of EEG microstates-associations with modalities of thinking. Neuroimage 125:643-656

Milz P, Pascual-Marqui RD, Achermann P, Kochi K, Faber PL (2017) The EEG microstate topography is predominantly determined by intracortical sources in the alpha band. Neuroimage 162:353-361

Morikawa T, Hayashi M, Hori T (1997) Auto power and coherence analysis of delta-theta band EEG during the waking-sleeping transition period. Electroencephalogr Clin Neurophysiol 103:633-641

Murray MM, Brunet D, Michel CM (2008) Topographic ERP analyses: a step-by-step tutorial review. Brain Topogr 20:249-264

Muthukrishnan SP, Ahuja N, Mehta N, Sharma R (2016) Functional brain microstate predicts the outcome in a visuospatial working memory task. Behav Brain Res 314:134-142. https://doi.org/10.1016/j. bbr.2016.08.020

Niedermeyer E (2005a) The normal EEG of the waking adult. In: Niedermeyer E, Da Silva LF (eds) Electroencephalography: basic principles, clinical applications, and related fields. Lippincott Williams \& Wilkins, Philadelphia

Niedermeyer E (2005b) Sleep and EEG. In: Niedermeyer E, Da Silva LF (eds) Electroencephalography: basic principles, clinical applications, and related fields. Lippincott Williams \& Wilkins, Philadelphia

Nishida K, Morishima Y, Yoshimura M, Isotani T, Irisawa S, Jann K, Dierks T, Strik W, Kinoshita T, Koenig T (2013) EEG microstates associated with salience and frontoparietal networks in frontotemporal dementia, schizophrenia and Alzheimer's disease. Clin Neurophysiol 124:1106-1114

Noreika V, Kamke MR, Canales-Johnson A, Chennu S, Mattingley JB, Bekinschtein TA (2017) Neurobehavioral dynamics of drowsiness. bioRxiv. https://doi.org/10.1101/155754

Ogilvie RD (2001) The process of falling asleep. Sleep Med Rev 5:247-270

Ogilvie RD, Wilkinson RT (1984) The detection of sleep onset: behavioral and physiological convergence. Psychophysiology 21:510-520

Overgaard M, Overgaard R (2011) Measurements of consciousness in the vegetative state. Lancet 378:2052-2054

Pascual-Marqui RD, Lehmann D, Faber P, Milz P, Kochi K, Yoshimura M, Nishida K, Isotani T, Kinoshita T (2014) The resting microstate networks (RMN): cortical distributions, dynamics, and frequency specific information flow. http://arxiv.org/abs/1212.6573v1

Pasqual-Marqui RD, Michel CM, Lehmann D (1995) Segmentation of brain electrical activity into microstates: model estimation and validation. IEEE Trans Biomed Eng 42:658-665

Platt JC (1999) Probabilistic outputs for support vector machines and comparisons to regular likelihood methods. Adv Large Margin Classif 10:61-74

Prerau MJ, Hartnack KE, Obregon-Henao G, Sampson A, Merlino M, Gannon K, Bianchi MT, Ellenbogen JM, Purdon PL (2014)
Tracking the sleep onset process: an empirical model of behavioral and physiological dynamics. PLoS Comput Biol 10:e1003866

Purdon PL, Pierce ET, Mukamel E, Prerau MJ, Walsh JL, Wong KFK, Salazar-Gomez AF, Harrell PG, Sampson AL, Cimenser A, Ching S, Kopell NJ, Tavares-Stoeckel C, Habeeb K, Merhar R, Brown EN (2013) Electroencephalogram signatures of loss and recovery of consciousness from propofol. Proc Natl Acad Sci USA 110:E1142-E1151

Sanders RD, Tononi G, Laureys S, Sleigh J (2013) Unconsciousness, not equal to unresponsiveness. Anesthesiology 116:946-959

Schlegel F, Lehmann D, Faber PL, Milz P, Gianotti LRR (2012) EEG microstates during resting represent personality differences. Brain Topogr 25:20-26

Seitzman BA, Abell M, Bartley SC, Erickson MA, Bolbecker AR, Hetrick WP (2016) Cognitive manipulation of brain electric microstates. Neuroimage 146:533-543

Steriade M, McCormick D, Sejnowski T (1993) Thalamocortical oscillations in the sleeping and aroused brain. Science 262:679-685

Storey JD (2002) A direct approach approach to false discovery rates. J R Stat Soc 64:479-498

Strelets V, Faber PL, Golikova J, Novototsky-Vlasov V, Koenig T, Gianotti LRR, Gruzelier JH, Lehmann D (2003) Chronic schizophrenics with positive symptomatology have shortened EEG microstate durations. Clin Neurophysiol 114:2043-2051

Šušmáková K, Krakovská a (2007) Classification of waking, sleep onset and deep sleep by single measures. Meas Sci Rev 7:34-38

Tagliazucchi E, Laufs H (2014) Decoding wakefulness levels from typical fMRI resting-state data reveals reliable drifts between wakefulness and sleep. Neuron 82:695-708. https://doi.org/10.1016/j.neuro n.2014.03.020

Tanaka H, Hayashi M, Hori T (1997) Topographical characteristics and principal component structure of the hypnagogic EEG. Sleep 20:523-534

Tanaka H, Hayashi M, Hori T (1998) Topographic mapping of electroencephalography coherence in hypnagogic state. Psychiatry Clin Neurosci 52:147-148

Tanaka H, Hayashi M, Hori T (2000) Topographical characteristics of slow wave activities during the transition from wakefulness to sleep. Clin Neurophysiol 111:417-427

Tomescu MI et al (2014a) Deviant dynamics of EEG resting state pattern in 22q11.2 deletion syndrome adolescents: a vulnerability marker of schizophrenia? Schizophr Res 157:175-181. https://doi. org/10.1016/j.schres.2014.05.036

Tomescu MI, Rihs Ta, Becker R, Britz J, Custo A, Grouiller F, Schneider M, Debbané M, Eliez S, Michel CM (2014b) Deviant dynamics of EEG resting state pattern in 22q11.2 deletion syndrome adolescents: a vulnerability marker of schizophrenia? Schizophr Res 157:175-181

Tomescu MI et al (2018) From swing to cane: sex differences of EEG resting-state temporal patterns during maturation and aging. Dev Cogn Neurosci 31:58-66. https://doi.org/10.1016/j.den.2018.04.011

Tukey JW (1949) Comparing individual means in the analysis of variance. Biometrics 5:99-114

Van de Ville D, Britz J, Michel CM (2010) EEG microstate sequences in healthy humans at rest reveal scale-free dynamics. Proc Natl Acad Sci USA 107:18179-18184

Vanhaudenhuyse A et al (2010) Two distinct neuronal networks mediate the awareness of environment and of self. J Cog Neurosci 23:570 578. https://doi.org/10.1162/jocn.2010.21488

Vanhaudenhuyse A, Demertzi A, Schabus M, Noirhomme Q, Bredart S, Boly M, Phillips C, Soddu A, Luxen A, Moonen G, Laureys S (2011) Two distinct neuronal networks mediate the awareness of environment and of self. J Cogn Neurosci 23:570-578

Vert J, Tsuda K, Schölkopf B (2004) A primer on kernel methods. Kernel Methods Comput Biol 47:35-70 
Vidaurre D, Quinn AJ, Baker AP, Dupret D, Tejero-Cantero A, Woolrich MW (2016) Spectrally resolved fast transient brain states in electrophysiological data. Neuroimage 126:81-95

Vinck M, Oostenveld R, van Wingerden M, Battaglia F, Pennartz CMA (2011) An improved index of phase-synchronization for electrophysiological data in the presence of volume-conduction, noise and sample-size bias. Neuroimage 55:1548-1565

Wright KP, Badia P, Wauquier A (1995) Topographical and temporal patterns of brain activity during the transition from wakefulness to sleep. Sleep 18:880-889 